\title{
Effect of magnetic field on imbibition phenomenon in fluid flow through fractured porous media with different porous material
}

https://doi.org/10.1515/nleng-2018-0059

Received March 23, 2018; revised June 20, 2018; accepted August 3, 2018.

\begin{abstract}
In this paper, the counter-current Imbibition phenomenon is considered in a fractured heterogeneous porous medium with the consideration of different porous materials like fine sand, volcanic sand and glass beads and magnetic field effect. The Homotopy analysis method is used here to derive an expression for finding the saturation profiles in a fractured heterogeneous porous medium with and without considering the magnetic field effect. Simulation results are developed for the saturation profiles to study the effect of inclination and the viscosity variation of native fluids on the saturation rate and the recovery rate of the reservoir with some interesting choices of parameters.
\end{abstract}

Keywords: Magnetic fluids, Homotopy analysis method, Fractured heterogeneous porous media, Counter- current Imbibition, Nonlinear, Partial differential equation

\section{Introduction}

Imbibition phenomenon is a process in which some fluid filled in porous medium comes in to contact with other fluid by which the solid is being preferentially wetted, as a result the wetting fluid will flow spontaneously through the solid walls of the pores into the medium and on the other side the residual fluid will be expelled. Imbibition created due to the differences in the wetting abilities of the fluid is called counter-current imbibition and it is a natural process that depends upon the porous medium as well as on the injection rate in a reservoir. In recent years, the

\footnotetext{
V. P. Gohil, Department of Applied Mathematics and Humanities, Sardar Vallabhbhai National Institute of Technology Surat, Gujarat, India, E-mail:vpgohil0909@gmail.com

*Corresponding Author: Ramakanta Meher, Department of Applied Mathematics and Humanities, Sardar Vallabhbhai National Institute of Technology Surat, Gujarat, India, E-mail: meher_ramakanta@yahoo.com
}

study of different effects, namely magnetic field (due to the magnetic fluid particles involved in injected water), viscosity, homogeneity, heterogeneity, fractured, porosity, capillary pressure and relative permeability on oil recovery system is a most challenging problem in the field of petroleum engineering and hydrogeology.

Many researchers have taken their keen interest in studying the different types of phenomena that arises in fluid flow through fractured porous media. Countercurrent imbibition phenomenon is an important phenomenon that has been discussed by many researchers from different point of view. Verma [25] discussed analytically a case of water-oil imbibition phenomena in a cracked porous medium with the consideration of a bar of finite length through perturbation approach. Similarly Verma [26] and Reis and Cil [19] developed a mathematical model for imbibition and fingering phenomena on ground water replenishment in a cracked porous media. Babadagli[3] studied the effects of temperature on the efficiency ofthe capillary imbibition mechanism and concluded that due to the temperature variation and increment in the capillary imbibition rate results a reduction in viscosity and interfacial tension. Standnes [22] experimentally studied the effect of boundary conditions on oil recovery in presence of co- and counter-current spontaneous imbibition.Tavassoli and Zimmerman [23] studied this phenomena in a strongly water-wet system and derived an expression for the saturation profiles through an approximate analytical approach withthe consideration of viscosity of the native fluid.Behbani et al.[5] derived an expression for the saturation profile in counter-current imbibition phenomena which shows that the behavior of twodimensional displacement can be predicted by using a one-dimensional model.While Ruth et.al [20] analyzed the same phenomena in a semi-infinite porous medium with the consideration of capillary pressure and relative permeability as a unique function of saturation which is independent of the nature of displacement and time. Meher and Mehta [12] considered capillary pressure and studied this phenomena in a homogenous porous media analytically through an exponential self-similar solutions tech- 
nique.Patel and Meher [15-17] considered fractured media, inclination and gravitational effect and explored the same phenomenon to decide the saturation rate and the recovery rate of the reservoir through a semi analytic approach in a heterogeneous porous media. Gohil and Meher [9] used homotopy analysis method and considered the same phenomenon for a time positive fractional kind to study the anomalous behaviour of the saturation of wetting phase.Vu [28] developed a theoretical model for imbibition phenomena in fluid flow through anisotropic fractured porous media and simulated this model through boundary element method. Abbasi et al. [1] considered the gravitational effects in counter-current imbibition phenomena while Liang et al. [10] discussed the development in oil-water saturation rate in spontaneous imbibition with the help of Nuclear Magnetic Resonance. De Borst [8] studied the fluid flow in a fractured porous media by using extended finite element method (FEM).

Patel and Meher $[15,16]$ considered fractured, inclination and gravitational effect and studied this phenomenon with the consideration of two porous materials in a heterogeneous porous media. In this paper, the work of Patel and Meher $[15,16]$ has been extended to a fractured heterogeneous porous media with the consideration of viscosity and magnetic field effect with three porous material namely fine sand, volcanic sand and glass beads. The novelty part of this study is to study the effects of viscosity of native fluid and the magnetic field on saturation rate in deriving the expression for finding the saturation rate and the recovery rate of the reservoir with the presence of different porous materials.

\section{Mathematical formulation of the problem}

Here it is considered a cylindrical block of length ' $L$ ' having it's all sides are surrounded by a leak proof surface except one open end. Imbibition surface is labelled by face $x=0$ and assumed that the cylindrical block be inclined at an angle $\alpha$ with the ground surface as shown in figures 1 and 2. It is also assumed that the capillary pressure of wetting phase is low, having a thin layer of magnetic field on the surface due to the presence of magnetic fluid particles in injected fluids. Due to the low capillary pressure of wetting phase, the counter current imbibition exist on the line of the interface.

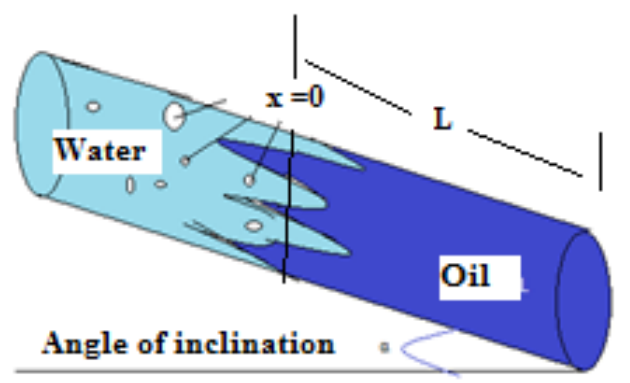

Fig. 1: Inclination effect in counter-current imbibition phenomenon

\section{Imbibition surface}

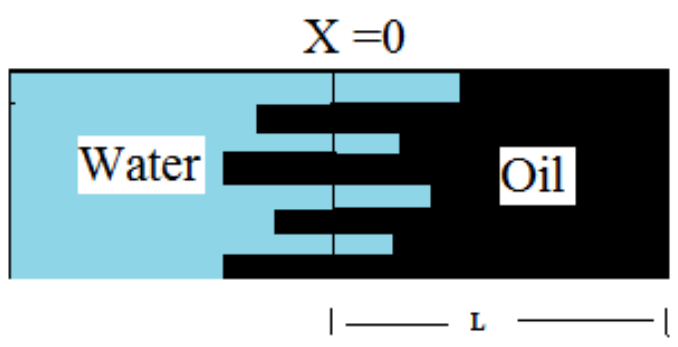

Fig. 2: Schematic diagram of imbibition phenomenon

The equation describing the conservation of mass [16, 21] for oil \& water is

$$
\frac{\partial}{\partial t}\left(\varphi(x) S_{i} \rho_{i}\right)+\nabla \cdot\left(\rho_{i} v_{i}\right)-\rho_{i} q_{i}=0
$$

Neglecting the compressibility of fluids (i.e. $\rho_{i}^{\prime} s$ of oil and water are constant), the conservation Eq. (1) reduces to the following form

$$
\varphi(x) \frac{\partial}{\partial t}\left(S_{i}\right)+\nabla \cdot\left(v_{i}\right)-q_{i}=0
$$

Since the problem is dealing with one dimensional form only, the Eq. (2) becomes

$$
\varphi(x) \frac{\partial S_{i}}{\partial t}+\frac{\partial v_{i}}{\partial x}-q_{i}=0
$$

where $v_{i}$ is the seepage velocity of water and oil which can be expressed by using Darcy's law as

$$
v_{i}=-K(x) \frac{k_{i}}{\mu_{i}}\left(\nabla \cdot p_{i}-g \rho_{i} \sin \alpha\right)
$$

In linear case, Eq. (4) becomes

$$
v_{i}=-K(x) \frac{k_{i}}{\mu_{i}}\left(\frac{\partial p_{i}}{\partial x}-g \rho_{i} \sin \alpha\right) \text { where } i=w, o
$$

Since the problem is dealing with an external effect of magnetic field, so an additional pressure that is exerted due to the pressure of a layer of magnetic fluid 
in the displacing fluid (w) [27] can be expressed as [26] $\left[\mu_{m} \lambda+\frac{16 \pi \mu_{m} \lambda^{2} r^{3}}{9(l+2)^{2}}\right] H \frac{\partial H}{\partial x}$

Hence the equations of seepage velocity (5) of water and oil with magnetic fluid particles becomes

$$
\begin{gathered}
v_{w}=-K(x) \frac{K_{w}}{\mu_{w}}\left[\frac{\partial p_{w}}{\partial x}+y H \frac{\partial H}{\partial x}-\rho_{w} g \sin \alpha\right] \\
v_{o}=-K(x) \frac{K_{o}}{\mu_{o}}\left(\frac{\partial p_{o}}{\partial x}-\rho_{o} g \sin \alpha\right) .
\end{gathered}
$$

where

$$
y=\left[\mu_{0} \lambda+\frac{16 \pi \mu_{0} \lambda^{2} r^{3}}{9(l+2)^{2}}\right]
$$

Since the problem is dealing with counter-current imbibition phenomenon in heterogeneous porous media, so the relation between the seepage velocity of oil and water, permeability and porosity of the porous medium can be expressed as [12]

$$
\begin{gathered}
v_{w}=-v_{o} \\
\varphi=\varphi(x)=\frac{1}{a-b x}, a-b x \geq 1, K=K_{C} \phi(x)
\end{gathered}
$$

The definition of capillary pressure gives

$$
p_{c}=p_{o}-p_{w}
$$

Eqs. (6), (7) and (9) together gives

$$
\frac{\partial p_{w}}{\partial x}=\frac{-\left(\frac{k_{o}}{\mu_{o}} \frac{\partial p_{c}}{\partial x}+\frac{k_{w}}{\mu_{w}} y H \frac{\partial H}{\partial x}-\frac{k_{o} \rho_{o}}{\mu_{o}} g \sin \alpha-\frac{k_{w} \rho_{w}}{\mu_{w}} g \sin \alpha\right)}{\left(\frac{k_{w}}{\mu_{w}}+\frac{k_{o}}{\mu_{o}}\right)}
$$

which gives

$$
v_{w}=K \frac{k_{w} k_{o}}{\mu_{w}}\left[\frac{\frac{\partial p_{c}}{\partial x}-y H \frac{\partial H}{\partial x}+\left(\rho_{w}-\rho_{o}\right) g \sin \alpha}{P^{\star} k_{w}+k_{o}}\right]
$$

Where $P^{\star}=\frac{\mu_{o}}{\mu_{w}}$

Hence Eq. (3) reduces to

$$
\begin{aligned}
& \phi(x) \frac{\partial S_{w}}{\partial t}+\frac{\partial}{\partial x}\left(K \frac{k_{w} k_{o}}{\mu_{w}}\left[\frac{\frac{\partial p_{c}}{\partial x}-y H \frac{\partial H}{\partial x}+\left(\rho_{w}-\rho_{o}\right) g \sin \alpha}{P^{\star} k_{w}+k_{o}}\right]\right) \\
& -q_{w}=0
\end{aligned}
$$

As per Brooks-Corey model [6, 16], the relationship of capillary pressure, relative permeability \& phase saturation for counter-current imbibition phenomena are

$$
\begin{gathered}
p_{c}\left(S_{w}\right)=p_{d}\left(\frac{S_{w}-S_{w r}}{1-S_{w r}}\right)^{\frac{-1}{\lambda}} q_{w}(x, t)=\frac{A}{c x+d} \\
k_{w}=\left(\frac{S_{w}-S_{w r}}{1-S_{w r}}\right)^{\frac{2+3 \lambda}{\lambda}}
\end{gathered}
$$

$$
k_{o}=\left(\frac{1-S_{w}}{1-S_{w r}}\right)^{2}\left(1-\left(\frac{S_{w}-S_{w r}}{1-S_{w r}}\right)^{\frac{2+\lambda}{\lambda}}\right)
$$

Where $S_{w r}$ is the residual saturation of wetting phase.

The magnetic field intensity [27] $H$ is only in X-direction so it can be expressed as [26]

$$
H=\frac{\Lambda}{x},
$$

Where $\Lambda$ is Magnetic field intensity constant Using Eqs. (14) to (17) in Eq. (13), it gives

$$
\begin{aligned}
& \phi(x) \frac{\partial S_{w}}{\partial t}-\frac{K_{C} p_{d}}{\lambda \mu_{w}} \frac{\partial}{\partial x}\left(\phi S_{w}^{e}\left(\frac{1}{1-S_{w r}}\right) \frac{\partial S_{w}}{\partial x}\right) \\
& -\frac{y K_{C}}{\mu_{w}} \frac{\partial}{\partial x}\left(\phi S_{w}^{e} \frac{\Lambda}{x}\left(\frac{-\Lambda}{x^{2}}\right)\right) \\
& +\frac{K_{C}\left(\rho_{w}-\rho_{o}\right) g \sin \alpha}{\mu_{w}} \frac{\partial}{\partial x}\left(\phi S_{w}^{e}\right)=0
\end{aligned}
$$

Where

$$
S_{w}^{e}=\frac{\left(\frac{S_{w}-S_{w r}}{1-S_{w r}}\right)^{\frac{1+2 \lambda}{\lambda}}\left(\frac{1-S_{w}}{1-S_{w r}}\right)^{2}\left(1-\left(\frac{S_{w}-S_{w r}}{1-S_{w r}}\right)^{\frac{2+\lambda}{\lambda}}\right)}{P^{\star}\left(\frac{S_{w}-S_{w r}}{1-S_{w r}}\right)^{\frac{2+3 \lambda}{\lambda}}+\left(\frac{1-S_{w}}{1-S_{w r}}\right)^{2}\left(1-\left(\frac{S_{w}-S_{w r}}{1-S_{w r}}\right)^{\frac{2+\lambda}{\lambda}}\right)}
$$

By using the dimensionless variables

$$
X=\frac{x}{L}, T=\frac{K_{c} y \Lambda^{2}}{\mu_{w} L^{4}} t
$$

and using the simplification of Eq. (10), it gives

$$
\frac{1}{\phi} \frac{\partial \phi}{\partial x}=\frac{b}{a} L
$$

The dimensionless form of Eq. (19) takes the form

$$
\begin{aligned}
& \frac{\partial S_{w}}{\partial T}-C_{0} \frac{\partial}{\partial X}\left(S_{w}^{e}\left(\frac{1}{1-S_{w r}}\right) \frac{\partial S_{w}}{\partial X}\right) \\
& -C_{0} \frac{b L^{2}}{a}\left(S_{w}^{e}\left(\frac{1}{1-S_{w r}}\right) \frac{\partial S_{w}}{\partial X}\right)-\frac{\partial}{\partial X}\left(S_{w}^{e} \frac{1}{X^{3}}\right) \\
& -\frac{b L^{2}}{a}\left(S_{w}^{e} \frac{1}{X^{3}}\right)+C_{1} \frac{\partial}{\partial X}\left(S_{w}^{e}\right)+C_{1} \frac{b L^{2}}{a}\left(S_{w}^{e}\right) \\
& -C_{2}\left(\frac{a-b_{1} x}{c_{1} x+d}\right)=0
\end{aligned}
$$

where, $\quad C_{0}=\frac{L^{2} p_{d}}{\Lambda^{2} y \lambda}$ and $C_{1}=\frac{g L^{3}\left(\rho_{w}-\rho_{o}\right) \sin \alpha}{\Lambda^{2} y}$

With appropriate initial condition

$$
S_{w}(X, 0)=e^{-X} \text {, with, } 0 \leq X \leq 1
$$


By considering the negligible effect of the magnetic field in imbibition phenomena, the Eq. (19) reduces to

$$
\begin{aligned}
& \frac{\partial S_{w}}{\partial t}-\frac{K_{C} p_{d}}{\lambda \mu_{w}} \frac{\partial}{\partial x}\left(S_{w}^{e}\left(\frac{1}{1-S_{w r}}\right) \frac{\partial S_{w}}{\partial x}\right) \\
& -\frac{K_{C} p_{d} b L}{\lambda \mu_{w} a}\left(S_{w}^{e}\left(\frac{1}{1-S_{w r}}\right) \frac{\partial S_{w}}{\partial x}\right) \\
& +\frac{K_{C}\left(\rho_{w}-\rho_{o}\right) g \sin \alpha}{\mu_{w}} \frac{\partial}{\partial x}\left(S_{w}^{e}\right) \\
& +\frac{K_{C}\left(\rho_{w}-\rho_{o}\right) g \sin \alpha b L}{\mu_{w} a}\left(S_{w}^{e}\right)-\frac{A}{(c x+d)}=0
\end{aligned}
$$

Again by introducing $X=\frac{x}{L}$ and $T=\frac{K_{C} p_{d}}{\lambda \mu_{w} L^{2}} t$ (dimensionless variables) in the Eq. (25) , the dimensionless form of Eq. (25) takes the form

$$
\begin{aligned}
& \frac{\partial S_{w}}{\partial T}-\frac{\partial}{\partial X}\left(S_{w}^{e}\left(\frac{1}{1-S_{w r}}\right) \frac{\partial S_{w}}{\partial X}\right) \\
& -\frac{b L^{2}}{a}\left(S_{w}^{e}\left(\frac{1}{1-S_{w r}}\right) \frac{\partial S_{w}}{\partial X}\right)+C \frac{\partial}{\partial X}\left(S_{w}^{e}\right) \\
& +C \frac{b L^{2}}{a}\left(S_{w}^{e}\right)-C^{\prime} \frac{\left(a-b_{1} X\right)}{\left(c_{1} X+d\right)}=0
\end{aligned}
$$

Where $C=\frac{\lambda g L\left(\rho_{w}-\rho_{o}\right) \sin \alpha}{p_{d}}, C^{\prime}=\frac{\lambda \mu_{w} L^{2} A}{k_{c} p_{d}}$

\section{Solution through homotopy analysis method}

The initial approximation through HAM [11] is

$$
S_{w, 0}=e^{-X}
$$

Using HAM, the linear and nonlinear terms for the solution of Eq. (22) can be defined as

$$
L(\phi(X, T ; q))=\frac{\partial \phi(X, T ; q)}{\partial T}
$$

$N(\phi(X, T ; q))=\frac{\partial \phi(X, T ; q)}{\partial t}-C_{0} \frac{\partial}{\partial X}\left(S_{w}^{\star}\left(\frac{1}{1-S_{w r}}\right) \frac{\partial \phi(X, T ; q)}{\partial X}\right)$ $-C_{0} \frac{b L^{2}}{a}\left(S_{w}^{\star}\left(\frac{1}{1-S_{w r}}\right) \frac{\partial \phi(X, T ; q)}{\partial X}\right)-\frac{\partial}{\partial X}\left(\Phi \frac{1}{X^{3}}\right)-\frac{b L^{2}}{a}\left(S_{w}^{\star} \frac{1}{X^{3}}\right)$ $\left.+C_{1} \frac{\partial}{\partial X}\left(S_{w}^{\star}\right)+C_{1} \frac{b L^{2}}{a}\left(S_{w}^{\star}\right)\right)-C_{2} \frac{\left(a-b_{1} X\right)}{\left(c_{1} X+d\right)}=0$

Where $S_{w}^{*}=\frac{\left(\frac{\phi(X, T ; q)-S_{w r}}{1-S_{w r}}\right)^{\frac{2+3 \lambda}{\lambda}}\left(\frac{1-\phi(X, T ; q)}{1-S_{w r}}\right)^{2}\left(1-\left(\frac{\phi(X, T ; q)-S_{w r}}{1-S_{w r}}\right)^{\frac{2+\lambda}{\lambda}}\right)}{P^{*}\left(\frac{\phi(X, T ; q)-S_{w r}}{1-S_{w r}}\right)^{\frac{2+3 \lambda}{\lambda}}+\left(\frac{1-\phi(X, T ; q)}{1-S_{w r}}\right)^{2}\left(1-\left(\frac{\phi(X, T ; q)-S_{w r}}{1-S_{w r}}\right)^{\frac{2+\lambda}{\lambda}}\right)}$

From the definition the so-called zero ${ }^{\text {th }}$ order deformation equation can be constructed as

$$
(1-q) L\left[\phi(X, T ; q)-S_{w, 0}(X, T)\right]=q h N(\phi(X, T ; q))
$$

When $q=0$ and $q=1$, Eq. (30) reduces to $\phi(X, T ; 0)=$ $S_{w, 0}(X, T)$ and $\phi(X, T ; 1)=S_{w}(X, T)$ respectively.

Expanding $\phi(X, T ; q)$ in Taylor series with respect to $q$,

$$
\varphi(X, T ; q)=S_{w, 0}(X, T)+\sum_{m=1}^{\infty} S_{w, m}(X, T) q^{m}
$$

Where

$$
S_{w, m}(X, T)=\left.\frac{1}{m !} \frac{\partial^{m} \varphi(X, T ; q)}{\partial q^{m}}\right|_{q=0}
$$

The convergence of the series (31) depends upon the auxiliary parameter. If it is convergent at $q=1$, one has

$$
S_{w}(X, T)=S_{w, 0}(X, T)+\sum_{m=1}^{\infty} S_{w, m}(X, T)
$$

Which must be one of the solutions of the original nonlinear equation, as proven by Liao [27].

Using the zero ${ }^{\text {th }}$ order deformation equation the $\mathrm{m}^{\text {th }}$ order deformation equation can be constructed as

$$
L\left[S_{w, m}(X, T)-\chi_{m} S_{w, m-1}(X, T)\right]=h R_{m}\left(\overrightarrow{S_{m-1}}\right)
$$

Using the inverse operator in Eq. (33), which gives

$$
S_{w, m}(X, T)=\chi_{m} S_{w, m-1}(X, T)+h L^{-1}\left[R_{m}\left(\overrightarrow{S_{m-1}}\right)\right]
$$

Hence, the approximate analytical solution of Eq. (22) corrected up to two approximations is

$$
\begin{aligned}
& S_{w}=e^{-X}+h T\left[C_{0} \frac{S_{w}{ }^{*} e^{-X}}{1-S_{w r}}+\frac{C_{1} b L^{2} S_{w}{ }^{* *}}{a}-\frac{S_{w}{ }^{* \star}}{X^{4}}\right. \\
& +\frac{2 S_{w}{ }^{* \star} e^{-X}}{X^{3}\left(1-S_{w r}\right)}+\frac{b L^{2} S_{w}{ }^{* \star}}{a X^{3}}+\frac{(2+\lambda) S_{w}{ }^{* \star}\left(S_{w r}{ }^{*}\right)^{2} \lambda e^{-X}}{X^{3}\left(1-S_{w r}\right) \lambda}-\frac{(2+3 \lambda) S_{w}{ }^{* \star} e^{-X}}{X^{3}\left(e^{-X}-S_{w r}\right) \lambda} \\
& -\frac{S_{w}{ }^{\star \star} S_{w}{ }^{\star \star \star}}{X^{3}}-C_{0}\left\{-\frac{2 S_{w}{ }^{*} e^{-X}}{\left(1-S_{w r}\right)\left(1-e^{-X}\right)}+\frac{S_{w}{ }^{*} e^{-X}}{1-S_{w r}}-\frac{(2+\lambda) S_{w}{ }^{*}\left(S_{w r}{ }^{*}\right)^{2} \lambda_{e^{-2 X}}}{\left(1-S_{w r}\right)^{2} \lambda}\right. \\
& \left.+\frac{(1+2 \lambda) S_{w}{ }^{*}\left(S_{w r}{ }^{*}\right)^{-1} / \lambda e^{-2 X}}{\left(1-S_{w r}\right)^{2} \lambda}+e^{-X} S_{w}{ }^{\star} S_{w}{ }^{\star \star \star}\right\}+C_{0}\left\{\frac{S_{w}{ }^{* \star} e^{-X}}{1-S_{w r}}\right. \\
& \left.-\frac{(2+\lambda) S_{w}{ }^{*}\left(S_{w r}{ }^{*}\right)^{2} \lambda e^{-X}}{\left(1-S_{w r}\right) \lambda}+\frac{(2+3 \lambda) S_{w}{ }^{* \star} e^{-X}}{\left(e^{-X}-S_{w r}\right)}-S_{w}{ }^{\star \star} S_{w}{ }^{\star \star \star}\right\}-C_{2} \frac{\left(a-b_{1} X\right)}{\left(c_{1} X+d\right)}
\end{aligned}
$$

Similarly, the approximate solution of Eq. (26) corrected up to two approximations is

$$
\begin{aligned}
& S_{w}=e^{-X}+h T\left[\frac{2 S_{w}^{*} e^{-X}}{\left(1-S_{w r}\right)\left(1-e^{-X}\right)}-\frac{S_{w}^{*} e^{-X}}{1-S_{w r}}+\frac{b S_{w}^{*}}{a\left(1-S_{w r}\right)}+\frac{b C S_{w}^{* *}}{a}\right. \\
& +\frac{(2+\lambda) S_{w}^{*}\left(S_{w r}^{*}\right)}{X^{3}\left(1-S_{w r}\right)^{2} \lambda}-\frac{(1+2 \lambda) S_{w}^{*} e^{-X}}{\left(e^{-X}-S_{w r}\right)\left(1-S_{w r}\right) \lambda}+\frac{e^{-X} S_{w}^{*} S_{w}^{* *}}{\left(1-S_{w r}\right)} \\
& \left.\left.+C\left\{\frac{2 S_{w}^{* *} e^{-X}}{\left(1-S_{w r}\right)}+\frac{(2+\lambda) S_{w}^{* *}\left(S_{w r}^{*}\right) \lambda e^{-X}}{\left(1-S_{w r}\right) \lambda}-\frac{(2+3 \lambda) S_{w}^{* *} e^{-X}}{\left(e^{-X}-S_{w r}\right)}--S_{w}^{\star *} S_{w}^{\star \star *}\right\}\right]\right) \\
& -C_{2} \frac{\left(a-b_{1} X\right)}{\left(c_{1} X+d\right)}
\end{aligned}
$$




$$
\begin{aligned}
\text { Where } S_{w r}^{\star}=\left(\frac{e^{-X}-S_{w r}}{1-S_{w r}}\right) \\
S_{w}^{\star}=\frac{\left(\frac{1-e^{-X}}{1-S_{w r}}\right)^{2}\left(1-\left(\frac{e^{-X}-S_{w r}}{1-S_{w r}}\right)^{\frac{2+\lambda}{\lambda}}\right)\left(\frac{e^{-X}-S_{w r}}{1-S_{w r}}\right)^{\frac{1+2 \lambda}{\lambda}}}{\left(\left(\frac{1-e^{-X}}{1-S_{w r}}\right)^{2}\left(1-\left(\frac{e^{-X}-S_{w r}}{1-S_{w r}}\right)^{\frac{2+\lambda}{\lambda}}\right)+P^{\star}\left(\frac{e^{-X}-S_{w r}}{1-S_{w r}}\right)^{\frac{2+3 \lambda}{\lambda}}\right)} \\
S_{w}^{\star \star}=\frac{\left(\frac{1-e^{-X}}{1-S_{w r}}\right)^{2}\left(1-\left(\frac{e^{-X}-S_{w r}}{1-S_{w r}}\right)^{\frac{2+\lambda}{\lambda}}\right)\left(\frac{e^{-X}-S_{w r}}{1-S_{w r}}\right)^{\frac{2+3 \lambda}{\lambda}}}{\left(\left(\frac{1-e^{-X}}{1-S_{w r}}\right)^{2}\left(1-\left(\frac{e^{-X}-S_{w r}}{1-S_{w r}}\right)^{\frac{2+\lambda}{\lambda}}\right)+P^{\star}\left(\frac{e^{-X}-S_{w r}}{1-S_{w r}}\right)^{\frac{2+3 \lambda}{\lambda}}\right)} \\
\quad S_{w}^{\star \star \star}=2 e^{-X}\left(\frac{1-e^{-X}}{1-S_{w r}}\right)^{2}\left(1-\left(\frac{e^{-X}-S_{w r}}{1-S_{w r}}\right)^{\frac{2+\lambda}{\lambda}}\right) \\
+\frac{P^{\star} e^{-X}\left(\frac{e^{-X}-S_{w r}}{1-S_{w r}}\right)^{\frac{2+2 \lambda}{\lambda}}(2+3 \lambda)}{\lambda\left(1-S_{w r}\right)} \\
+\frac{2 e^{-X}\left(\frac{1-e^{-X}}{1-S_{w r}}\right)^{2}\left(1-\left(\frac{e^{-X}-S_{w r}}{1-S_{w r}}\right)^{\frac{2+\lambda}{\lambda}}\right)\left(\frac{e^{-X}-S_{w r}}{1-S_{w r}}\right)^{\frac{2}{\lambda}}}{\lambda\left(1-S_{w r}\right)}
\end{aligned}
$$

Eq. (35) discusses the saturation profiles of injected fluid during imbibition phenomena in a fractured hetrogenous porous media with magnetic field effect where as Eq.(36) discusses the saturation profiles of injected fluid during imbibition phenomena in a fractured hetrogenous porous media without considering the magnetic field effect.

\section{Convergence Analysis of the Homotopy Analysis Method}

The exact square residual error [24] of a nonlinear equation $N\left[S_{w}(X, T)\right]=0$ is

$$
\operatorname{Res}(h)=\int_{\Omega}\left(N\left[\sum_{K=0}^{M} S_{w, k}(X, T)\right]\right)^{2} d \Omega
$$

Where $\Omega$ is the region of interest and $\sum_{K=0}^{M} S_{w, k}(X, T)$ gives $M^{\text {th }}$ order approximation.

Obviously when $\operatorname{Res}(h) \rightarrow 0$, the homotopy series solution converges to the exact solution.

By using maxima-minima concept, we will have to find out the minimum value of $\operatorname{Res}(h)$ such that we get the proper value of convergence control parameter $h$.

$$
\frac{d \operatorname{Res}(h)}{d h}=0
$$

Convergence control parameter $h$ can be obtained by using Eqs. (37) and (38). We can also use $h$ - cureve to choose the proper value of controlling parameter $h$ such that series solution (32) converges to the exact solution of given nonlinear problem as per following theorems 1 and 2

Theorem 1. Suppose that $A \subset R$ be a Banach space denoted with suitable norm, over which the sequence $S_{w, n}(X, T)$ is defined for some specified value of $h$. Assume that the initial approximation $S_{w, 0}(X, T)$ remains inside the ball of the solution $S_{w}(X, T)$. Taking $r \in R$ be a constant, the following statement hold true: If $\left\|S_{K+1}(X, T)\right\| \leq$ $r\left\|S_{K}(X, T)\right\|$ for all $K$, some $0<r<1$, the series (32) converges absolutely.

\section{Proof}

If $S_{n}^{\prime}(X, T)$ denote the sequence of partial sum of series (32). Now, we will prove that $S_{n}^{\prime}(X, T)$ is Cauchy sequence in $A$.

$$
\begin{aligned}
\left\|S_{n+1}^{\prime}(X, T)-S_{n}^{\prime}(X, T)\right\| & =\left\|S_{n+1}(X, T)\right\| \\
& \leq r\left\|S_{n}(X, T)\right\| \\
& \leq r^{2}\left\|S_{n-1}(X, T)\right\| \\
& \cdots \cdots \cdots . . . \\
& \leq r^{n+1}\left\|S_{0}(X, T)\right\|
\end{aligned}
$$

Now for every $m, n \in N$ and $n>m$,

$$
\begin{aligned}
\left\|S_{n}^{\prime}(X, T)-S_{m}^{\prime}(X, T)\right\|= & \| S_{n}^{\prime}(X, T)-S_{n-1}^{\prime}(X, T)+S_{n-1}^{\prime}(X, T) \\
- & S_{n-2}^{\prime}(X, T)+\ldots+S_{m+1}^{\prime}(X, T) \\
& -S_{m}^{\prime}(X, T) \| \\
\leq & \frac{1-r^{n-m}}{1-r} r^{m+1}\left\|S_{0}(X, T)\right\|
\end{aligned}
$$

Since $0<r<1$,

$$
\lim _{m, n \rightarrow \infty}\left\|S_{n}^{\prime}-S_{m}^{\prime}\right\|=0
$$

$\left\{S_{n}^{\prime}\right\}$ is Cauchy sequence in Bananch space so it is convergent.

Theorem 2. If the series solution defined in (32) is convergent, it converges to an exact solution of nonlinear problem (26). [11]

\section{Numerical results \& discussion}

\subsection{Effect of Viscosity on saturation rate}

Figure 3 discusses the effects of viscosity on saturation rate in different porous materials with and without considering 


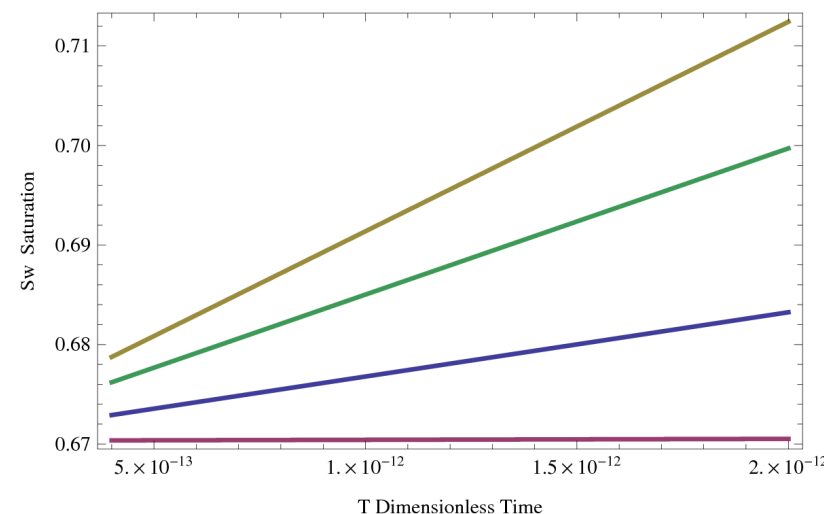

(a)

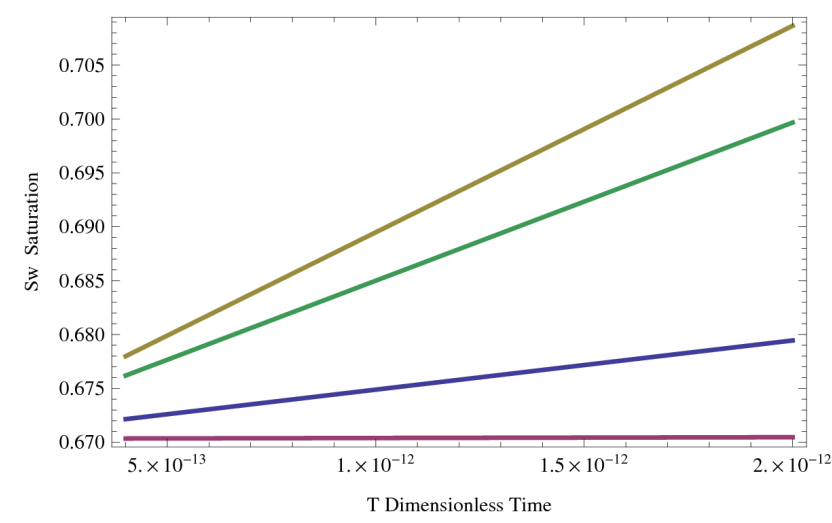

(c)

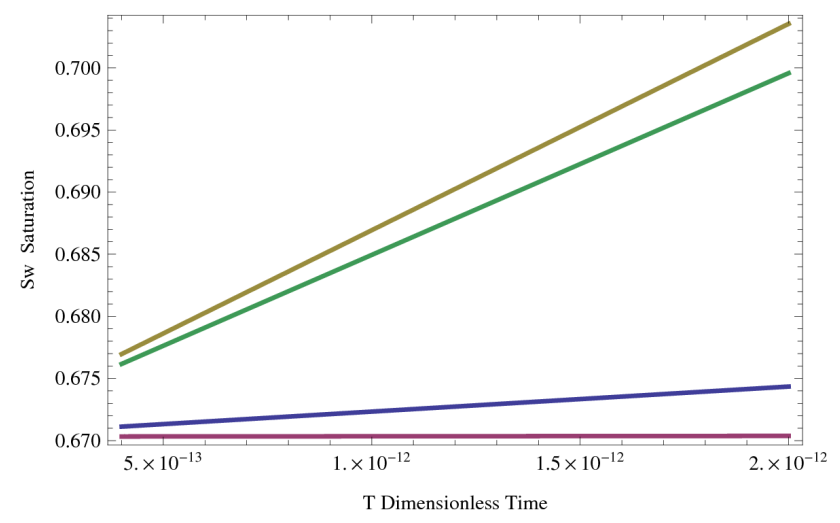

(e)

$$
\begin{aligned}
& \text { - Low Viscous Oil in matrix porous media } \\
& \text { - High Viscous Oil in matrix porous media }
\end{aligned}
$$

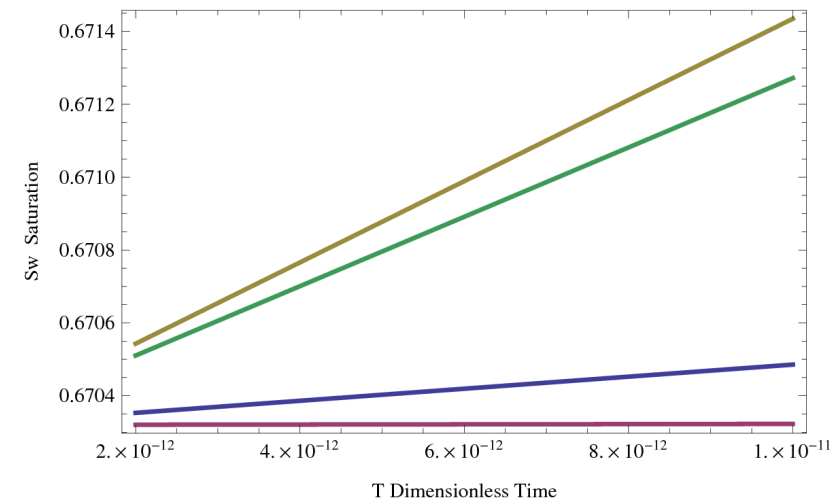

(b)

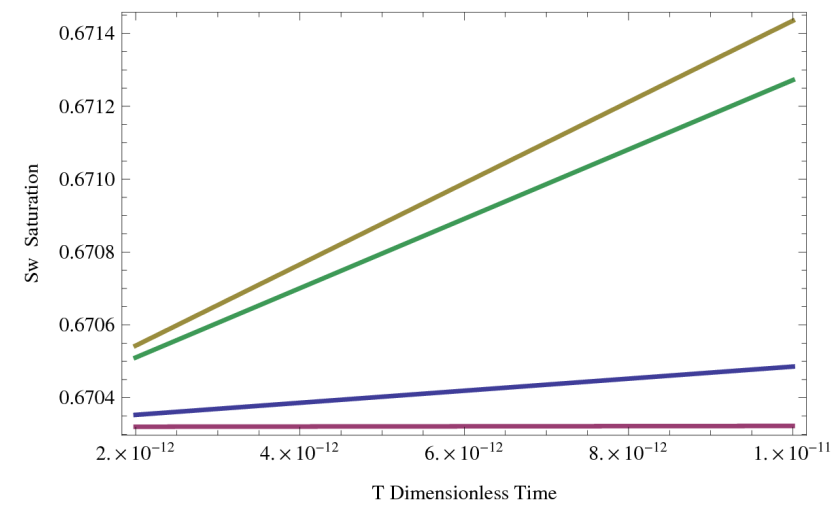

(d)

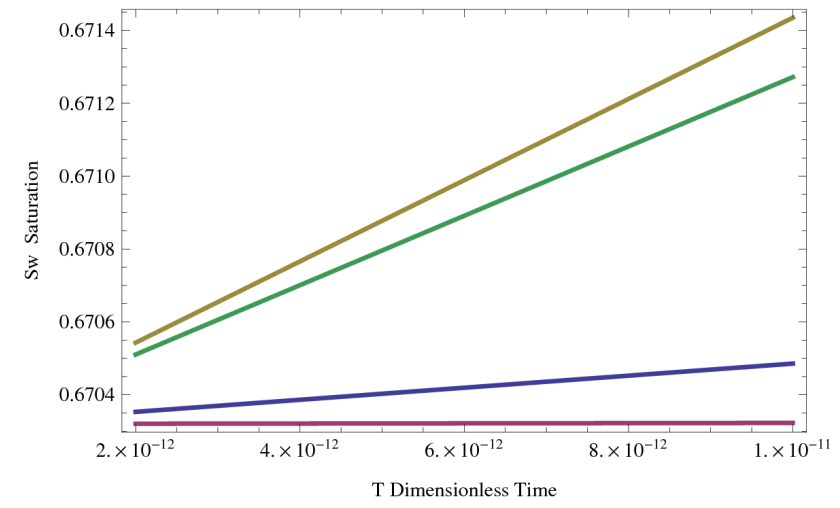

(f)

$$
\begin{aligned}
& \text { - Low Viscous Oil in fractured porous media } \\
& \text { - High Viscous Oil in fircurued porous modia }
\end{aligned}
$$

Fig. 3: Satuartion rate for Fine Sand (a) with magnetic field effect (b) without magnetic field effect,Satuartion rate for Volcanic Sand (c) with magnetic field effect (d) without magnetic field effect,Satuartion rate for Glass beads (e) with magnetic field effect (f) without magnetic field effect 
Table 1: Numerical values of saturation rate in volcanic sand in fractured and matrix porous media having low and high viscous reservoir fluids with and without magnetic field effect

\begin{tabular}{|c|c|c|c|c|c|c|c|c|}
\hline \multirow{3}{*}{ Time in Years } & \multicolumn{4}{|c|}{ Matrix Porous Medium } & \multicolumn{4}{|c|}{ Fractured Porous Medium } \\
\hline & \multicolumn{2}{|c|}{$\begin{array}{l}\text { With effect of } \\
\text { magnetic field }\end{array}$} & \multicolumn{2}{|c|}{$\begin{array}{l}\text { Without effect of } \\
\text { magnetic field }\end{array}$} & \multicolumn{2}{|c|}{$\begin{array}{l}\text { With effect of } \\
\text { magnetic field }\end{array}$} & \multicolumn{2}{|c|}{$\begin{array}{l}\text { Without effect of } \\
\text { magnetic field }\end{array}$} \\
\hline & $\begin{array}{c}\text { Oil with } \\
\text { low } \\
\text { viscosity }\end{array}$ & $\begin{array}{l}\text { Oil with } \\
\text { high } \\
\text { viscosity }\end{array}$ & $\begin{array}{c}\text { Oil with } \\
\text { low } \\
\text { viscosity }\end{array}$ & $\begin{array}{l}\text { Oil with } \\
\text { high } \\
\text { viscosity }\end{array}$ & $\begin{array}{l}\text { Oil with } \\
\text { low } \\
\text { viscosity }\end{array}$ & $\begin{array}{l}\text { Oil with } \\
\text { high } \\
\text { viscosity }\end{array}$ & $\begin{array}{c}\text { Oil with } \\
\text { low } \\
\text { viscosity }\end{array}$ & $\begin{array}{l}\text { Oil with } \\
\text { high } \\
\text { viscosity }\end{array}$ \\
\hline 14.24 & 0.672145 & 0.670349 & 0.670353 & 0.670321 & 0.677985 & 0.676189 & 0.670543 & 0.670510 \\
\hline 28.98 & 0.673970 & 0.670379 & 0.670386 & 0.670321 & 0.685649 & 0.682058 & 0.670766 & 0.670701 \\
\hline 42.72 & 0.675795 & 0.670408 & 0.670419 & 0.670322 & 0.693314 & 0.687927 & 0.670989 & 0.670891 \\
\hline 56.96 & 0.677620 & 0.670438 & 0.670452 & 0.670322 & 0.700978 & 0.693796 & 0.671212 & 0.671082 \\
\hline 71.20 & 0.679446 & 0.670468 & 0.670486 & 0.670323 & 0.708643 & 0.699666 & 0.671435 & 0.671272 \\
\hline
\end{tabular}

Table 2: Numerical values of saturation rate in fine sand in fractured and matrix porous media having low and high viscous reservoir fluids with and without magnetic field effect

\begin{tabular}{|c|c|c|c|c|c|c|c|c|}
\hline \multirow{3}{*}{ Time in Years } & \multicolumn{4}{|c|}{ Matrix Porous Medium } & \multicolumn{4}{|c|}{ Fractured Porous Medium } \\
\hline & \multicolumn{2}{|c|}{$\begin{array}{l}\text { With effect of } \\
\text { magnetic field }\end{array}$} & \multicolumn{2}{|c|}{$\begin{array}{l}\text { Without effect of } \\
\text { magnetic field }\end{array}$} & \multicolumn{2}{|c|}{$\begin{array}{l}\text { With effect of } \\
\text { magnetic field }\end{array}$} & \multicolumn{2}{|c|}{$\begin{array}{l}\text { Without effect of } \\
\text { magnetic field }\end{array}$} \\
\hline & $\begin{array}{l}\text { Oil with } \\
\text { low } \\
\text { viscosity }\end{array}$ & $\begin{array}{l}\text { Oil with } \\
\text { high } \\
\text { viscosity }\end{array}$ & $\begin{array}{l}\text { Oil with } \\
\text { low } \\
\text { viscosity }\end{array}$ & $\begin{array}{l}\text { Oil with } \\
\text { high } \\
\text { viscosity }\end{array}$ & $\begin{array}{l}\text { Oil with } \\
\text { low } \\
\text { viscosity }\end{array}$ & $\begin{array}{l}\text { Oil with } \\
\text { high } \\
\text { viscosity }\end{array}$ & $\begin{array}{l}\text { Oil with } \\
\text { low } \\
\text { viscosity }\end{array}$ & $\begin{array}{c}\text { Oil with } \\
\text { high } \\
\text { viscosity }\end{array}$ \\
\hline 14.24 & 0.672906 & 0.670358 & 0.670350 & 0.670321 & 0.678743 & 0.676198 & 0.670540 & 0.670510 \\
\hline 28.98 & 0.673549 & 0.670397 & 0.670381 & 0.670321 & 0.687166 & 0.682076 & 0.670761 & 0.670701 \\
\hline 42.72 & 0.678071 & 0.670435 & 0.670412 & 0.670322 & 0.695589 & 0.687954 & 0.670982 & 0.670891 \\
\hline 56.96 & 0.680654 & 0.670474 & 0.670443 & 0.670322 & 0.704012 & 0.693833 & 0.671203 & 0.671081 \\
\hline 71.20 & 0.683238 & 0.670513 & 0.670474 & 0.670323 & 0.712436 & 0.699711 & 0.671424 & 0.671272 \\
\hline
\end{tabular}

Table 3: Numerical values of saturation rate in glass beads in fractured and matrix porous media having low and high viscous reservoir fluids with and without magnetic field effect

\begin{tabular}{|c|c|c|c|c|c|c|c|c|}
\hline \multirow{3}{*}{$\begin{array}{l}\text { Time } \\
\text { in } \\
\text { Years } \\
-\end{array}$} & \multicolumn{4}{|c|}{ Matrix Porous Medium } & \multicolumn{4}{|c|}{ Fractured Porous Medium } \\
\hline & \multicolumn{2}{|c|}{$\begin{array}{l}\text { With effect of } \\
\text { magnetic field }\end{array}$} & \multicolumn{2}{|c|}{$\begin{array}{l}\text { Without effect of } \\
\text { magnetic field }\end{array}$} & \multicolumn{2}{|c|}{$\begin{array}{l}\text { With effect of } \\
\text { magnetic field }\end{array}$} & \multicolumn{2}{|c|}{$\begin{array}{l}\text { Without effect of } \\
\text { magnetic field }\end{array}$} \\
\hline & $\begin{array}{l}\text { Oil with } \\
\text { low } \\
\text { viscosity }\end{array}$ & $\begin{array}{l}\text { Oil with } \\
\text { high } \\
\text { viscosity }\end{array}$ & $\begin{array}{l}\text { Oil with } \\
\text { low } \\
\text { viscosity }\end{array}$ & $\begin{array}{l}\text { Oil with } \\
\text { high } \\
\text { viscosity }\end{array}$ & $\begin{array}{l}\text { Oil with } \\
\text { low } \\
\text { viscosity }\end{array}$ & $\begin{array}{l}\text { Oil with } \\
\text { high } \\
\text { viscosity }\end{array}$ & $\begin{array}{l}\text { Oil with } \\
\text { low } \\
\text { viscosity }\end{array}$ & $\begin{array}{l}\text { Oil with } \\
\text { high } \\
\text { viscosity }\end{array}$ \\
\hline 14.24 & 0.671089 & 0.670325 & 0.670340 & 0.670320 & 0.676928 & 0.670395 & 0.670530 & 0.670510 \\
\hline 28.98 & 0.671857 & 0.670336 & 0.670360 & 0.670321 & 0.683536 & 0.670544 & 0.670740 & 0.670700 \\
\hline 42.72 & 0.672626 & 0.670346 & 0.670380 & 0.670321 & 0.690145 & 0.670693 & 0.670949 & 0.670891 \\
\hline 56.96 & 0.673394 & 0.670357 & 0.670400 & 0.670321 & 0.696753 & 0.670842 & 0.671158 & 0.671081 \\
\hline 71.20 & 0.674164 & 0.670368 & 0.670420 & 0.670322 & 0.703361 & 0.670991 & 0.671369 & 0.671271 \\
\hline
\end{tabular}

the magnetic field effects which shows that the saturation rate is higher for low viscosus native fluids in fractures as compared to matrix porous media with magnetic field effect for all kinds of porous materials. Saturation rate is also higher in fine sand having low viscous native fluid as com- pared to volcanic sand and glass beads in fractured porous media under the effects of magnetic field while the saturation rate is lower in glass beads with highly viscous native fluid in matrix porous media without any effect of magnetic field at a particular distance from the initial point. 


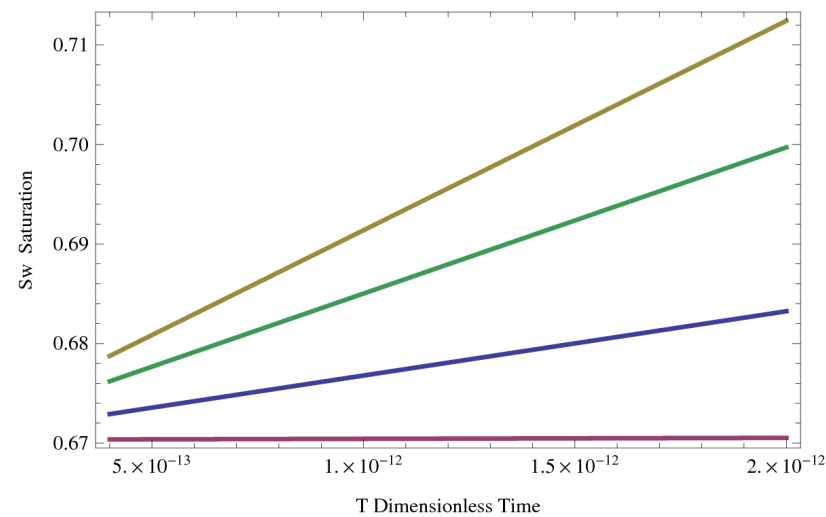

(a)

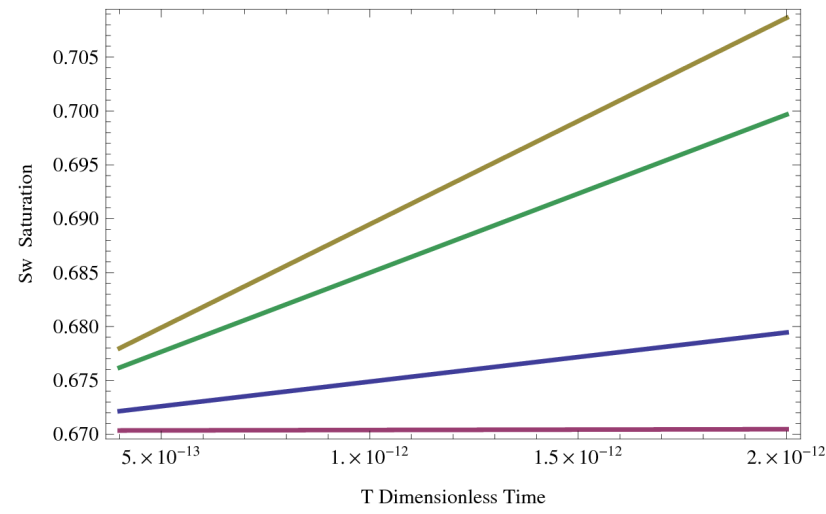

(c)

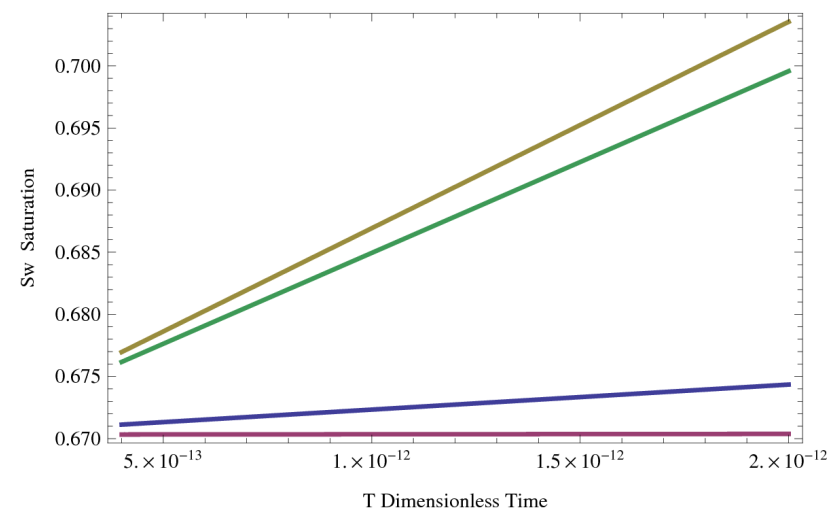

(e)

$$
\begin{aligned}
& \text { - Matrix porous media } \\
& \text { - Inclined matrx porous media }
\end{aligned}
$$

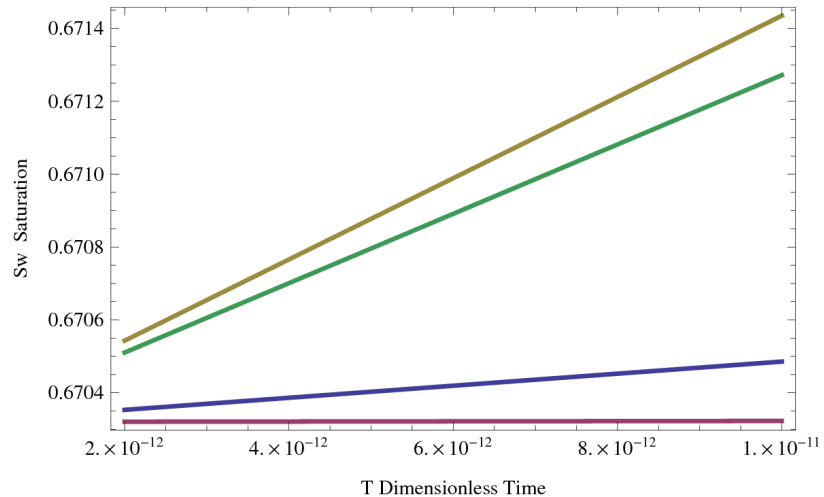

(b)

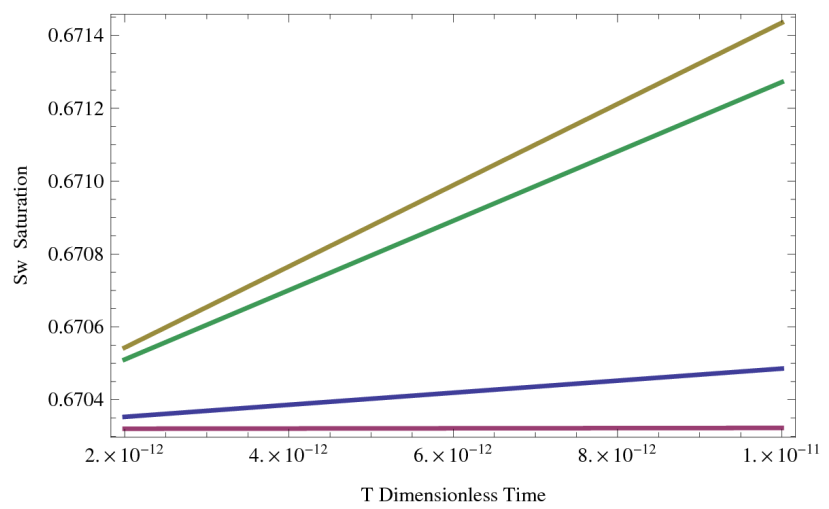

(d)

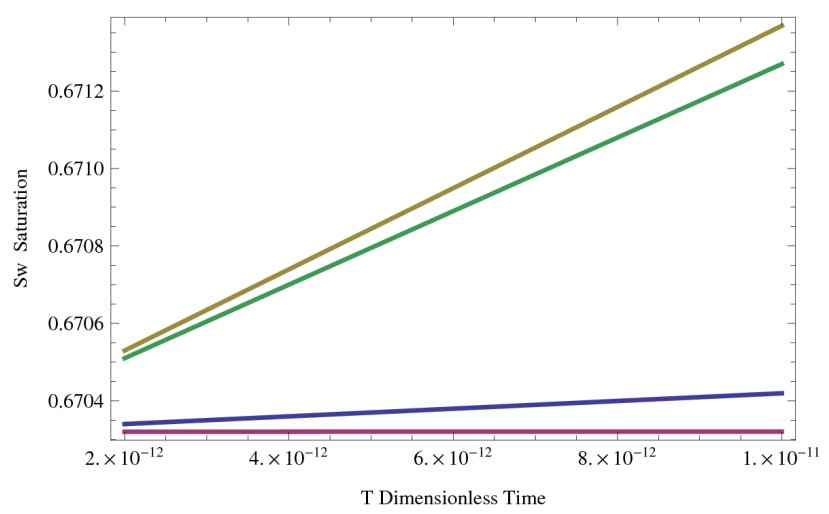

(f)

- Fractured porous media

— Inclined fractured porous media

Fig. 4: Satuartion rate for Fine Sand (a)with magnetic field effect (b) without magnetic field effect,Satuartion rate for Volcanic Sand (c)with magnetic field effect (d) without magnetic field effect,Satuartion rate for Glass beads (e)with magnetic field effect (f) without magnetic field effect 
Table 1,2 and 3 discusses the numerical values of saturation rate in different porous materials like Volcanic sand,Fine sand and glass beads having the effects of the viscosity of native fluid with and without considering the magnetic field effect in fractures as well as in porous matrix in porous media.

\subsection{Inclination effect on saturation rate}

Figure 4 discusses the effects of inclination on saturation rate which shows that there is an impact of inclination on the saturation rate in all kinds of porous materials. Saturation rate is higher in zero inclined plane as compared to higher inclined plane i.e the saturation rate is more for $\alpha=0^{\circ}$ in fractures and in fine sand with the effects of magnetic field. While the saturation rate is lower for $\alpha=10^{\circ}$ in glass beads in matrix porous medium without the effects of magnetic field and it implies as the angle of inclination of the plane be increases the saturation rate be decreases.

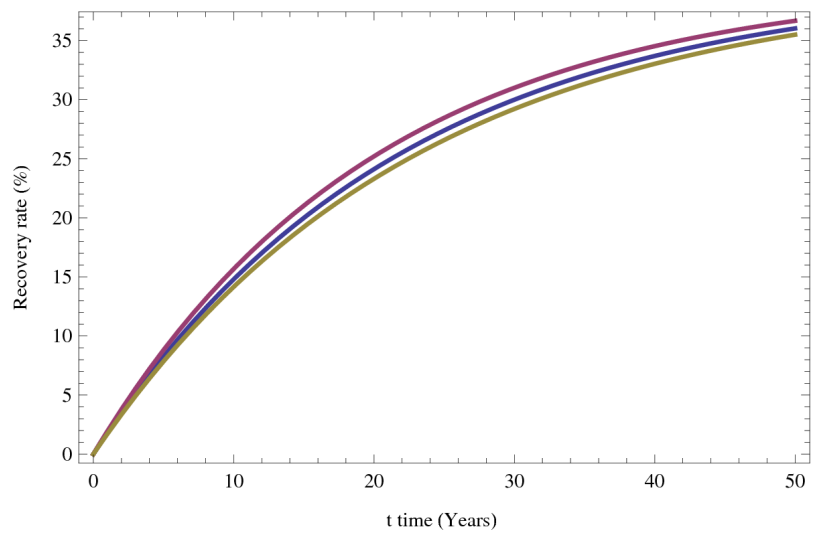

Fig. 5: Recovery rate in matrix porous medium under effect of magnetic field

Tables 4,5 and 6 discuss the numerical values of saturation rate in different kinds of porous materials in fractured and matrix porous media with consideration of inclination and magnetic field effect when the ground water and the native oil moves on an inclined plane inside the reservoir as shown in Fig. 1

\subsection{Recovery rate}

Oil recovery rate can be found by some specific proposed formula that matched with fluid properties and imbibition experiments. This formula has been used by Tavassoli et

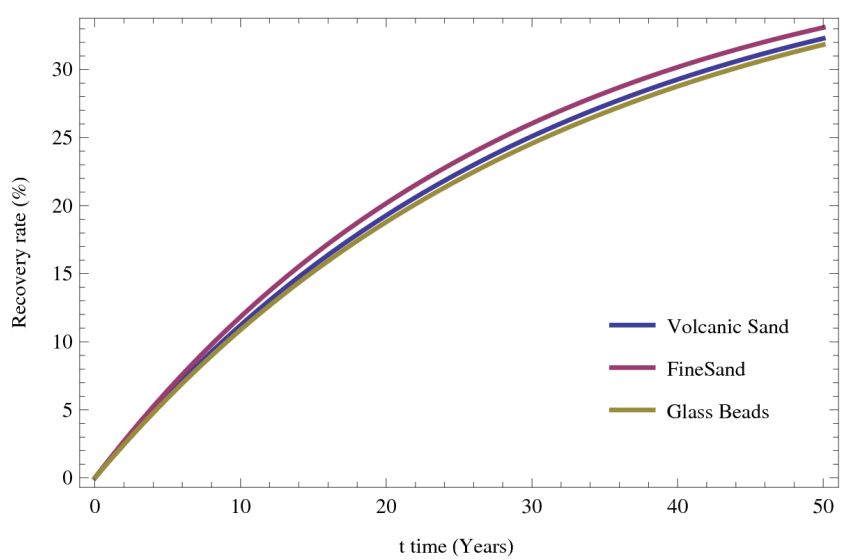

Fig. 6: Recovery rate in matrix porous medium without effect of magnetic field

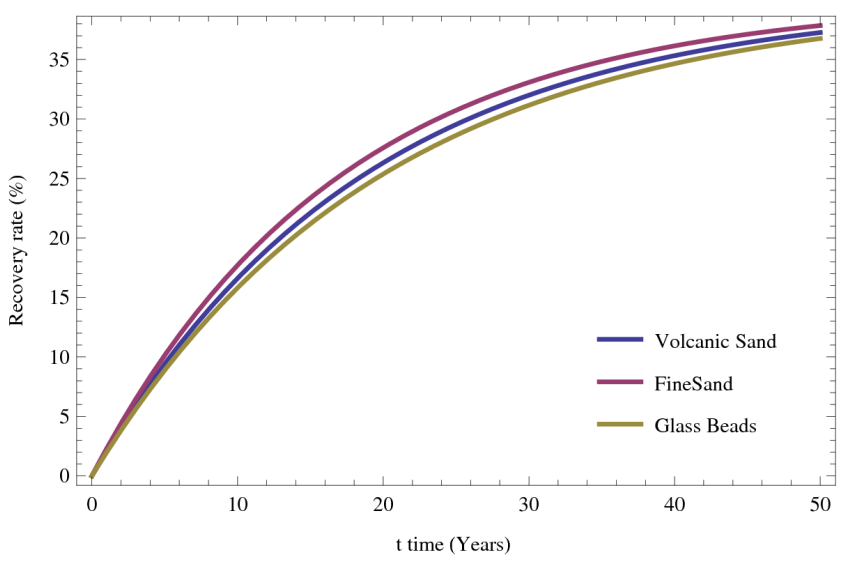

Fig. 7: Recovery rate in fractured porous medium under effect of magnetic field

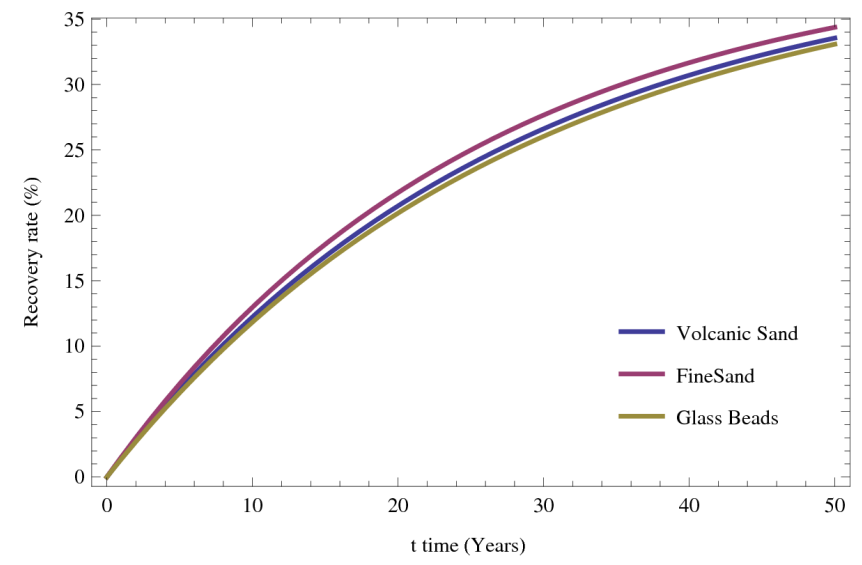

Fig. 8: Recovery rate in fractured porous medium without effect of magnetic field 
Table 4: Numerical values of saturation rate in volcanic sand in an inclined fractured and matrix porous medium with the effect of magnetic field

\begin{tabular}{|c|c|c|c|c|c|c|c|c|}
\hline \multirow{3}{*}{$\begin{array}{l}\text { Time } \\
\text { in } \\
\text { Years } \\
-\end{array}$} & \multicolumn{4}{|c|}{ Matrix Porous Medium } & \multicolumn{4}{|c|}{ Fractured Porous Medium } \\
\hline & \multicolumn{2}{|c|}{$\begin{array}{l}\text { With effect of } \\
\text { magnetic field }\end{array}$} & \multicolumn{2}{|c|}{$\begin{array}{l}\text { Without effect of } \\
\text { magnetic field }\end{array}$} & \multicolumn{2}{|c|}{$\begin{array}{l}\text { With effect of } \\
\text { magnetic field }\end{array}$} & \multicolumn{2}{|c|}{$\begin{array}{l}\text { Without effect of } \\
\text { magnetic field }\end{array}$} \\
\hline & $\alpha=\overline{0^{o}}$ & $\alpha=10^{\circ}$ & $\alpha=0^{o}$ & $\alpha=10^{\circ}$ & $\alpha=\overline{0^{o}}$ & $\alpha=10^{\circ}$ & $\alpha=0^{o}$ & $\alpha=10^{\circ}$ \\
\hline 4.24 & 0.672145 & 0.671678 & 0.670353 & 570320 & 677985 & 0.677517 & 0.670543 & 0.670510 \\
\hline 3.98 & 0.673970 & 0.673035 & 0.670386 & 0.670321 & 0.685649 & 0.6847 & 0.670766 & 0.670700 \\
\hline 2.72 & 0.675795 & 0.674393 & 0.670419 & 0.670321 & 0.693314 & 0.691912 & 0.670989 & 0.670890 \\
\hline 56.96 & 0.677620 & 0.675751 & 0.670452 & 0.670321 & 0.700978 & 0.699109 & 0.671212 & 0.671081 \\
\hline 1.20 & 0.679446 & 0.677109 & 0.670486 & 0.670322 & 0.708643 & 0.706307 & 0.671435 & 0.671271 \\
\hline
\end{tabular}

Table 5: Numerical values of saturation rate in fine sand in an inclined fractured and matrix porous medium with the effect of magnetic field

\begin{tabular}{|c|c|c|c|c|c|c|c|c|}
\hline \multirow{3}{*}{$\begin{array}{l}\overline{\text { Time }} \\
\text { in } \\
\text { Years } \\
-\end{array}$} & \multicolumn{4}{|c|}{ Matrix Porous Medium } & \multicolumn{4}{|c|}{ Fractured Porous Medium } \\
\hline & \multicolumn{2}{|c|}{$\begin{array}{l}\text { With effect of } \\
\text { magnetic field }\end{array}$} & \multicolumn{2}{|c|}{$\begin{array}{l}\text { Without effect of } \\
\text { magnetic field }\end{array}$} & \multicolumn{2}{|c|}{$\begin{array}{l}\text { With effect of } \\
\text { magnetic field }\end{array}$} & \multicolumn{2}{|c|}{$\begin{array}{l}\text { Without effect of } \\
\text { magnetic field }\end{array}$} \\
\hline & $\alpha=0^{o}$ & $\bar{\alpha}=10^{\circ}$ & $\alpha=\overline{0^{o}}$ & $\bar{\alpha}=10^{\circ}$ & $\alpha=\overline{0^{o}}$ & $\bar{\alpha}=10^{\circ}$ & $\alpha=\overline{0^{\circ}}$ & $\bar{\alpha}=10^{\circ}$ \\
\hline$\overline{14.24}$ & 0.672906 & 0.672440 & 670350 & .670320 & 0.678743 & 0.678280 & 0.670540 & 0.670510 \\
\hline 28.98 & 0.673549 & 0.674 & 0.670381 & 321 & 0.687166 & 0.686239 & 0.670761 & 0.6707 \\
\hline 42.72 & 0.678071 & 0.67 & 412 & 321 & 89 & 98 & 0.670982 & 0.67089 \\
\hline 56.96 & 0.680654 & 0.6788000 & 0.670443 & 321 & 0.704012 & 0.70 & 0.671203 & 0.671080 \\
\hline 1.20 & 0.683238 & 0.680920 & 0.670474 & 0.670322 & 0.712436 & 0.710118 & 0.671424 & 0.671270 \\
\hline
\end{tabular}

Table 6: Numerical values of saturation rate in glass beads and in an inclined fractured and matrix porous medium with the effect of magnetic field

\begin{tabular}{|c|c|c|c|c|c|c|c|c|}
\hline \multirow{3}{*}{$\begin{array}{l}\text { Time } \\
\text { in } \\
\text { Years } \\
-\end{array}$} & \multicolumn{4}{|c|}{ Matrix Porous Medium } & \multicolumn{4}{|c|}{ Fractured Porous Medium } \\
\hline & \multicolumn{2}{|c|}{$\begin{array}{l}\text { With effect of } \\
\text { magnetic field }\end{array}$} & \multicolumn{2}{|c|}{$\begin{array}{l}\text { Without effect of } \\
\text { magnetic field }\end{array}$} & \multicolumn{2}{|c|}{$\begin{array}{l}\text { With effect of } \\
\text { magnetic field }\end{array}$} & \multicolumn{2}{|c|}{$\begin{array}{l}\text { Without effect of } \\
\text { magnetic field }\end{array}$} \\
\hline & $\alpha=\overline{0^{o}}$ & $\bar{\alpha}=10^{\circ}$ & $\alpha=\overline{0^{\circ}}$ & $\alpha=10^{\circ}$ & $\alpha=\overline{0^{o}}$ & $\bar{\alpha}=10^{\circ}$ & $\alpha=0^{o}$ & $\bar{\alpha}=10^{\circ}$ \\
\hline 4.24 & 0.671089 & 0.670667 & 0.670340 & 0.670320 & 0.676928 & 0.676507 & 0.670530 & 0.670510 \\
\hline 8.98 & 0.671857 & 0.671015 & 0.670360 & 0.670320 & 0.683536 & 0.682694 & 0.670740 & 0.670700 \\
\hline 2.72 & 0.672626 & 0.671362 & 0.670380 & 0.670320 & 0.690145 & 0.688881 & 0.670949 & 0.670890 \\
\hline 6.96 & 0.673394 & 0.671710 & 0.670400 & 0.670320 & 0.696753 & 0.695068 & 0.671158 & 0.671080 \\
\hline 1.20 & 0.674164 & 0.672058 & 0.670420 & 0.670321 & 0.703361 & 0.701255 & 0.671369 & 0.671270 \\
\hline
\end{tabular}

al. [23], Patel and Meher [16, 17] in their previous research. For the first time, Aronofsky et al. [2] introduced the following function of recovery rate

$$
R=R_{\infty}\left(1-e^{-y T}\right)
$$

Here $R_{\infty}$ is the ultimate recovery that means the limit toward which the recovery converges, the recovery rate is $R$ and $y$ is a constant giving the rate of convergence.

Figure 5 to 9 discusses the variation in recovery rate in different porous materials in fractured and in matrix porous medium with and without considering magnetic field effect. From Table 7 and 8, it can be concluded that
$36.15 \%$ and $34.53 \%$ of the reservoir oil can be recovered in 40 years in fine sand with the effect of magnetic field. While in the same time duration $31.67 \%$ and $31.17 \%$ of oil can be recovered in fine sand without considering the effects of magnetic field in fractured porous media. Hence it reveals that the fractured fine sand is the best porous materials for the optimum recovery rate of the reservoir among all the three porous materials that has taken for comparison. Similarly from Fig. 5 to 9, it can also be concluded that the presence of magnetic field has a greater impact for an optimum recovery of the reservoir in all types of porous materials in fluid flow through fractured porous medium. 


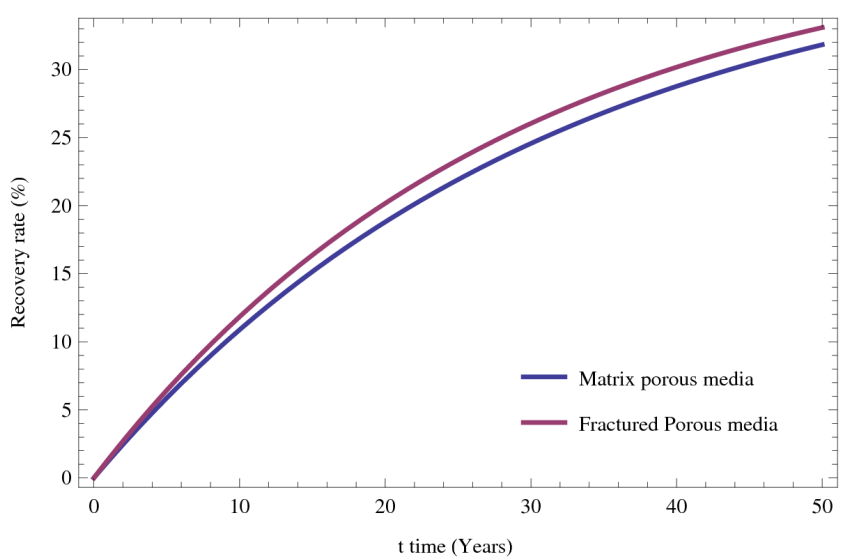

Fig. 9: Comparison of recovery rate in fractured and matrix porous medium

Table 7 and 8 discuss the numerical values of the recovery rate in fractured and in matrix porous medium in different porous materials with and without considering the magnetic field effect.

Table 7: Numerical values of recovery rate in various types of porous matrix

\begin{tabular}{|c|c|c|c|c|c|c|}
\hline \multirow{3}{*}{$\begin{array}{l}\text { Time } \\
\text { in } \\
\text { Years } \\
-\end{array}$} & \multicolumn{6}{|c|}{ Rate of oil recovery (\%) } \\
\hline & \multicolumn{3}{|c|}{$\begin{array}{l}\text { With effect of } \\
\text { magnetic field }\end{array}$} & \multicolumn{3}{|c|}{$\begin{array}{l}\text { Without effect of } \\
\text { magnetic field }\end{array}$} \\
\hline & $\begin{array}{c}\text { Volcanic } \\
\text { sand }\end{array}$ & $\begin{array}{l}\text { Fine } \\
\text { sand }\end{array}$ & $\begin{array}{l}\text { Glass } \\
\text { beads }\end{array}$ & $\begin{array}{c}\text { Volcanic } \\
\text { sand }\end{array}$ & $\begin{array}{l}\text { Fine } \\
\text { sand }\end{array}$ & $\begin{array}{l}\text { Glass } \\
\text { beads }\end{array}$ \\
\hline 0 & 0.00 & 0.00 & 0.00 & 0.00 & 0.00 & 0.00 \\
\hline 10 & 14.80 & 15.68 & 14.16 & 11.21 & 11.83 & 10.88 \\
\hline 20 & 24.12 & 25.21 & 30.00 & 19.28 & 20.17 & 18.80 \\
\hline 30 & 30.00 & 31.00 & 29.22 & 25.09 & 26.03 & 24.57 \\
\hline 40 & 33.70 & 34.53 & 33.04 & 29.27 & 31.17 & 28.77 \\
\hline
\end{tabular}

Table 8: Numerical values of recovery rate in various types of fractured porous medium

\begin{tabular}{|c|c|c|c|c|c|c|}
\hline \multirow{3}{*}{$\begin{array}{l}\text { Time } \\
\text { in } \\
\text { Years } \\
-\end{array}$} & \multicolumn{6}{|c|}{ Rate of oil recovery (\%) } \\
\hline & \multicolumn{3}{|c|}{$\begin{array}{l}\text { With effect of } \\
\text { magnetic field }\end{array}$} & \multicolumn{3}{|c|}{$\begin{array}{l}\text { Without effect of } \\
\text { magnetic field }\end{array}$} \\
\hline & $\begin{array}{l}\text { Volcanic } \\
\text { sand }\end{array}$ & $\begin{array}{l}\text { Fine } \\
\text { sand }\end{array}$ & $\begin{array}{l}\text { Glass } \\
\text { beads }\end{array}$ & $\begin{array}{c}\text { Volcanic } \\
\text { sand }\end{array}$ & $\begin{array}{l}\text { Fine } \\
\text { sand }\end{array}$ & $\begin{array}{l}\text { Glass } \\
\text { beads }\end{array}$ \\
\hline 0 & 0.00 & 0.00 & 0.00 & 0.00 & 0.00 & 0.00 \\
\hline 10 & 16.61 & 17.71 & 15.81 & 12.23 & 12.97 & 11.84 \\
\hline 20 & 26.32 & 27.58 & 25.37 & 20.72 & 21.74 & 20.17 \\
\hline 30 & 32.00 & 33.08 & 31.16 & 26.61 & 27.66 & 26.04 \\
\hline 40 & 35.32 & 36.15 & 34.65 & 30.71 & 31.67 & 30.17 \\
\hline
\end{tabular}

\section{Conclusion}

The significant part of the study is the study of the impact of viscous native fluid and magnetic field on saturation rate and on the recovery rate of the reservoir with the consideration of different porous materials during countercurrent imbibition phenomena in an inclined fractured heterogeneous porous media.It can be concluded that there is a greater impact of external force, porosity, permeability of different porous materials and the viscosity of native fluids on saturation rate and on the recovery rate of the reservoir. These results are very useful for petroleum reservoir engineers, researchers and geologist working in the field of petroleum reservoir problems.

\begin{tabular}{ccc}
\multicolumn{2}{c}{ Nomenclature } & \\
\hline Symbol & Unit & Quantity \\
$v$ & $m^{3} / s$ & Darcy's velocity of the phase \\
$\nabla$ & & Differential operator \\
$k_{i}$ & & Relative permeability of the $i^{\text {th }}$ phase \\
$K$ & $m^{2}$ & Permeability of the porous medium \\
$L$ & $m$ & Length \\
$p_{c}$ & $P a$ & Capillary pressure \\
$S$ & & Saturation of the phase \\
$\phi$ & & Porosity of soil \\
$\mu$ & $N s / m^{2}$ & Viscosity of fluid \\
$\rho$ & $k g / m^{3}$ & Density \\
$\beta$ & $N / m^{2}$ & Constant of Proportionality \\
$\chi$ & $m$ & Distance \\
$X$ & & Dimensionless variable of distance \\
$t$ & $s e c$ & time \\
$T$ & & Dimensionless variable of time \\
$S_{w}(x, t)$ & & Saturation of wetting phase \\
$g$ & $m / s^{2}$ & Acceleration due to gravity \\
$K_{c}$ & $m^{2}$ & Constant of proportionality \\
$a$ & & constant of variable porosity \\
$b$ & $m^{-1}$ & constant of variable porosity \\
$\alpha$ & & Inclination angle \\
$R$ & & Recovery \\
$R \infty$ & & Ultimate recovery \\
$S_{w r}$ & & Residual saturation of wetting phase \\
$\lambda$ & & Material grain size distribution \\
$q$ & & Source term
\end{tabular}

\section{References}

[1] Abbasi J, Riazi M, Ghaedi M. Modified shape factor incorporating gravity effects for scaling counter current imbibition. Journal of Petroleum Science and Engineering 2017, 150, 108- 
114.

[2] Aronofsky JS, Masse L, Natanson SG. A model for the mechanism of oil recovery from the porous matrix due to water invasion in fractured reservoirs, 1958.

[3] Babadagli T. Temperature effect on heavy-oil recovery by imbibition in fractured reservoirs. Journal of Petroleum Science and Engineering 1996, 4(3-4), 197-208.

[4] Bear J. Dynamic of fluid in porous media, American Elsevier Publishing Company, 1972.

[5] Behbahani HS, Donato GD, Blunt MJ. Simulation of countercurrent imbibition in water-wet fractured reservoirs. Journal of Petroleum Science and Engineering 2006, 50(1), 21-39.

[6] Brooks RH. Hydraulic properties of porous media. Colorado State University, Hydro Paper 3 (27), 1964.

[7] Chen Z. Reservoir simulation: mathematical techniques in oil recovery. Society for Industrial and Applied Mathematics, 2007.

[8] De Borst R. Fluid flow in fractured and fracturing porous media: A unified view. Mechanics Research Communications 2017, 80, 7-57.

[9] Gohil VP, Meher R. Homotopy Analysis Method for solving Counter current imbibition phenomena of the time positive fractional type arising in heterogeneous porous media. International Journal of Mathematics \& Computation 2017, 28(2), 77-85.

[10] Liang B, Jiang H, Li J et. al. Investigation of Oil Saturation Development behind Spontaneous Imbibition Front Using Nuclear Magnetic Resonance T2. Energy \& Fuels 2017, 31(1), 473481.

[11] Liao SJ. Beyond Perturbation: Introduction to the Homotopy Analysis Method. Chapman and Hall/CRC, 2004.

[12] Meher R, Mehta M and Meher S. Exponential self similar solutions technique for imbibition phenomenon arising in double phase flow through porous medium with capillary pressure. Int. J. of Appl. Math and Mech 2011, 7(8), 29-40.

[13] Patel HS, Meher R. Effect of Heterogeneity on Imbibition Phenomena in Fluid Flow through Porous Media with Different Porous Materials, Nonlinear Engineering, 2018. DOI: https://doi.org/10.1515/nleng-2017-0122

[14] Patel HS, Meher R. Modelling of imbibition phenomena in twophase fluid flow through fractured porous media, Nonlinear Engineering 2017, 6(1),15-24.

[15] Patel HS, Meher R. Simulation of Fingering Phenomena in Fluid Flow through Fractured Porous Media with Inclination and Gravitational Effect. Journal of Applied Fluid Mechanics 2016, 9(6), 3135-3145.
[16] Patel HS, Meher R. Simulation of Imbibition Phenomena in Fluid Flow through Fractured Heterogeneous Porous Media with Different Porous Materials. Journal of Applied Fluid Mechanics 2017, 10(5), 1451-1460.

[17] Patel HS, Meher R. Simulation of counter-current imbibition phenomenon in a double phase flow through fractured porous medium with capillary pressure 2017. Ain Shams Engineering Journal.

[18] Patel KR, Mehta MN, Patel TR. A mathematical model of imbibition phenomenon in heterogeneous porous media during secondary oil recovery process. Applied Mathematical Modelling 2013, 37(5), 2933-2942.

[19] Reis JC, Cil M. A model for oil expulsion by counter-current water imbibition in rocks: one-dimensional geometry. Journal of Petroleum Science and Engineering 1993,10(2),97-107

[20] Ruth DW, Li Y, Mason G, Morrow NR. An approximate analytical solution for counter-current spontaneous imbibition. Transport in Porous Media 2007, 66(3) , 373-390

[21] Scheidegger AE. The physics of flow through porous media.University of Toronto, 1974.

[22] Standnes DC. Experimental study of the Impact of boundary conditions on oil recovery by co-current and counter-current spontaneous imbibition. Energy Fuels 2004, 18, 271 - 282.

[23] Tavassoli Z, Zimmerman RW, Blunt MJ. Analytic analysis for oil recovery during counter-current imbibition in strongly waterwet systems. Transport in Porous Media 2005, 58, 173-189.

[24] Turkyilmazoglu M. An effective approach for evaluation of the optimal convergence control parameter in the homotopy analysis method. Filomat 2016, 30(6) 1633-1650.

[25] Verma AP. Imbibition in a cracked porous medium. Canadian journal of Physics 1969, 47(22), 2519-24.

[26] Verma AP. Fingero Imbibition in Artificial Replenishment of Ground Water Through Cracked Porous Medium. Water Resources Research 1970, 6(3), 906-11.

[27] Verma AP, Rajput AK. Instabilities in displacement processes through porous media with magnetic fluid. Journal of Magnetism and Magnetic Materials 1987, 65(2-3), 330-4.

[28] Vu MN, Nguyen ST, Vu MH. Modeling of fluid flow through fractured porous media by a single boundary integral equation. Engineering Analysis with Boundary Elements 2015, 59, 16671. 